\title{
Influences de la variabilité génétique et environnementale sur la digestibilité in vitro ou in vivo du maïs fourrage *
}

\author{
Y Barrière 1, C Demarquilly 2, Y Hebert 1, P Dardenne ${ }^{3}$, \\ J Andrieu 2, P Maupetit 4, M Lila 1, JC Émile 1 \\ ${ }^{1}$ INRA, station d'amélioration des plantes fourragères, 86600 Lusignan; \\ 2 INRA, station de recherches sur la nutrition des herbivores, 63122 Theix, France; \\ 3 Station de Haute Belgique, 600 Libramont; \\ 4 ITCF, 9170 Boigneville, Belgique
}

(Reçu le 21 juin 1990; accepté le 28 janvier 1991)

\begin{abstract}
Résumé - Dans le cadre d'une collaboration entre instituts de recherche, de développement et établissements de sélection, des travaux ont été entrepris pour préciser la pertinence de différentes mesures in vitro de la digestibilité du maïs fourrage, en comparaison à la mesure classique de référence faite par des moutons à l'entretien. Seize hybrides ont été répartis sur les 7 lieux susceptibles de réaliser les mesures in vivo, et les essais ont été répétés 2 années consécutives. Les différences entre génotypes pour le CUD** de la matière organique, mesuré par les moutons standards ne sont significatives que si l'on inclut un hybride atypique brown-midrib-3. En revanche, chacune des 3 méthodes de digestibilité in vitro testée conduit à des effets génotypes significatifs. Les corrélations entre mesures de digestibilité avec chacune des méthodes in vitro sont élevées et voisines de 0,9 , mais diminuent jusqu'à 0,6 si on limite l'étude aux fourrages dont la teneur en matière sèche est au moins égale à $27,5 \%$. Toutefois, ce sont les mêmes génotypes qui sont reconnus pour leur faible ou forte digestibilité par chacune des 3 méthodes, et les valeurs obtenues in vivo leur apparaissent faiblement liées puisque les corrélations entre digestibilité in vivo et in vitro varient de 0,5 à 0,1 . Les liaisons entre les constituants organiques mesurés (cellulose brute, NDF**, ADF**, lignine) et les différents critères de digestibilité in vitro sont moyennement élevées et négatives, comprises entre $-0,8$ et $-0,6$; elles sont plus faibles avec le CUD de la matière organique, entre $-0,5$ et $-0,3$ et voisines de 0,0 avec le CUD de la cellulose brute. Dans une optique de sélection de variétés de maïs fourrage améliorées pour leur digestibilité en plante entière, les résultats montrent que ces tests, que l'on peut étalonner dans de très bonnes conditions dans le proche infrarouge $(\mathrm{NIRS})^{* *}$, permettent au moins de répartir les génotypes en groupes distincts pour ce critère. Même si les liaisons entre ces critères in vitro et les valeurs établies avec les moutons standards sont faibles, les regroupements établis sur la base des valeurs in vitro ne sont pas en contradiction avec les valeurs obtenues sur moutons, en particulier pour les hybrides les moins digestibles. Ces outils in vitro et in vivo n'expriment pas les mêmes variations, digestibilité maximale potentielle d'une part, et valorisation par un animal à l'entretien d'autre part. De plus, ils diffèrent par la précision des mesures et la répartition de l'importance de chacun des effets pris en compte. La pertinence de ces tests in vitro devra en fait être mesurée en terme de capacité ou non à prédire une production de lait ou de viande chez des ruminants à haut potentiel, et des améliorations à ces techniques de tests pourront être apportées pour tenter d'intégrer l'aspect facilité de dégradation des constituants pariétaux.
\end{abstract}

Zea mays = maïs (ensilage) / digestibilité in vivo / digestibilité in vitro / solubilité enzymatique / mouton standard / variabilité génétique

Summary - Effect of genotypic and environmental variability on in vitro and in vivo digestibility of silage maize. Results on relevance and suitability of in vitro tests for digestibility improvement in maize are considered, and compared with standard measurements in sheep within a collaborative network between research institute, technical institutes and private breeding companies. Sixteen hybrids were dispatched to 7 locations fitted out for work with sheep in digestibility crates. Experiments were replicated 2 yr running. Six animals were fed for 5 or $6 d$ after a 10-d period of inurement. Forages were harvested 4-7 times after silking, and dry-matter content of forage offered varied

\footnotetext{
*Expérimentation réalisée par le «Club digestibilité»; liste des membres : INRA, CRA Gembloux, ITCF, AGPM, Limagrain, Asgrow France, Semences Cargill, Caussade semences, Ciba Geigy, France Canada semences SA, Pioneer France maïs, Maïsadour, Maïs angevin, Nickerson SA, Northrup King, RAGT SA, Rustica semences, SEM Diffusion, Recherche Euromaïs, SES France. ** CUD : coefficient d'utilisation digestive; NDF : Neutral detergent fiber, ADF : Acid detergent fiber, NIRS; E: Near infrared spectroscopy
} 
from $18.3-53.1 \%$, with an average value of $29.0 \%$. Genotype effects are significant for in vitro tests of digestibility and for in vivo digestibility of crude fiber, but in vivo digestibility of organic matter is significant only when a brown-midrib-3 (bm3) hydrid is included (table II). Phenotypic variability for in vivo digestibility of organic matter ranged from 71.0 to 74.2 and 78.5 for the bm3 hybrid, and respectively from 52.3 to 63.1 and 70.1 for in vivo digestibility of crude fiber (table IV). Correlations between the different digestibility tests are good when computed with all forage offered; they are quite low $(\approx 0.6)$ for classic forages, the dry matter content of which is higher or equal to $27.5 \%$ (table $V)$. But in any case, the same genotypes are ranked as low, or ranked as high with all in vitro digestibility measurements, and in vivo tests are consistent with these results. Correlations between in vitro tests and in vivo sheep tests of digestibility ranked from 0.6-0.1 when including or not a bm3 genotype, and when considering or not forage, dry content of which is higher or equal to $27.5 \%$. Correlations between biochemical constituents (crude fiber, NDF, ADF, lignin) and in vivo or in vitro estimates of digestibility are negative and fairly high $(-0.8$ to -0.5$)$, but null with crude fiber digestibility. It seems easy to calibrate the in vitro and in vivo tests under very good conditions with NIRS. For breeding silage maize with a higher whole plant digestibility, these results are promising because it seems possible when using in vitro tests to assign the hybrids into 2 or 3 groups for digestibility characteristics. Correlations with standard tests in sheep are mostly due to maturity stage, locations or year of experiment and standard deviations are higher in animal tests than with enzymatic of NIRS tests. Further investigation should now be carried out on the relevance of these in vitro tests in predicting the effect of genetic variability of silage maize on the milk yield of dairy cows, or on the average daily gain of young bulls or steers. The in vitro tests may also be improved to include cell wall digestibility susceptibility.

Zea mays : maize (silage) / in vivo digestibility / in vitro digestibility / enzymatic solubility / standard sheep / genetic variability

\section{INTRODUCTION}

A côté de critères agronomiques comme la productivité en plante entière et la résistance à la verse en végétation pour chacune des gammes de précocité, la valeur alimentaire pour des ruminants est un des critères importants de sélection du maïs destiné à une utilisation sous forme d'ensilage. Le sélectionneur doit pour cela disposer de méthodes d'estimation utilisables sans difficulté et à un coût raisonnable sur de grands effectifs. Dans cet esprit ont été reprises ou développées l'utilisation de méthodes de mesures in vitro de la digestibilité par solubilités enzymatiques (Aufrère, 1982; Lila et al, 1986; Ronsin et Femenias, 1990). D'un point de vue technique, ces différentes méthodes s'avèrent fiables et répétables, en particulier après étalonnage et prédiction par spectrométrie de réflectance dans le proche infrarouge (NIRS), (Biston et Dardenne, 1988). Toutefois, nous ne disposons pas encore de références en nombre suffisant pour juger de la pertinence de ces outils et ne savons donc pas relier une amélioration de la digestibilité in vitro du maïs à une amélioration, ou non, de la production de lait ou de viande. Intermédiaire entre les mesures in vitro et les valeurs effectivement observées avec des gros ruminants, la digestibilité mesurée sur moutons standards permet d'estimer la valeur énergétique de référence en UFL (Andrieu et al, 1988; Jarrige, 1988), qui est, avec les quantités ingérées, un des facteurs de la valeur alimentaire et des performances zootechniques. L'objectif de ce travail, réalisé dans le cadre d'une collaboration entre I'INRA, le CRA de Gembloux, I'ITCF, I'AGPM et un groupe d'établissements de sélection réunis dans le "Club digestibilité du maïs", est donc de contribuer à préciser la pertinence ou la valeur prédictrice des tests in vitro dont nous pouvons disposer, d'une part en étudiant la variabilité phénotypique pour des caractéristiques de valeur alimentaire, d'autre part en étudiant les liaisons entre les mesures réalisées in vitro et les valeurs obtenues sur moutons standards, et cela en intégrant des effets stade de maturité par des prélèvements successifs au cours de la période de maturation des plantes de maïs.

\section{MATÉRIELS ET MÉTHODES}

Seize hybrides ont été répartis sur 7 lieux d'expérimentation disposant d'ateliers de mesures de la digestibilité in vivo par des moutons en cage (tableau I).

Les hybrides sont des variétés inscrites au catalogue français, ou européen (variété 15), avec un génotype expérimental brown-midrib-3 produit par la station INRA de Lusignan (hybride 17, (F2bm3 $X$ $\mathrm{F} 16 \mathrm{bm} 3) \times \mathrm{F} 113 \mathrm{bm} 3$ ). Ils se répartissent dans les 5 groupes classiques de précocité, à savoir très précoces $(7,8,16)$, précoces $(2,10,12,15)$, demiprécoces $(1,3,4,11,13,17)$, demi-tardifs (5) et tardifs $(6,14)$. La répartition des hybrides sur les différents lieux résulte d'un compromis entre les contraintes liées au nombre de traitements possibles sur chacun de ces lieux, la nécessité de travailler sur une variabilité génétique quelque peu représentative des hybrides inscrits, le fait que les différentes conditions des mi- 
Tableau I. Répartition des 16 hybrides sur les 7 lieux d'expérimentation.

Hybrides

\author{
1 INRA Theix (63) \\ 2 INRA Rennes (35) \\ 3 INRA Lusignan (86) \\ 4 INRA Le Pin (61) \\ 5 INRA-ENSAA Dijon (21) \\ 6 ITCF La Jaillière (49) \\ 7 ENSAT Toulouse (31)
}

1
1
1
1
1

34

2

456
6
$\begin{array}{ll}7 & 8\end{array}$

2

2

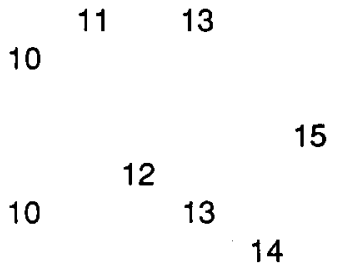

16 17 lieux imposaient de prendre en compte la précocité des hybrides, et les possibilités de traitement statistique des données. Les essais ont été répétés 2 années successives (1987-1988), mais les résultats obtenus à Toulouse la première année ont du être éliminés (moutons trop jeunes, en croissance et non à l'entretien), ainsi que ceux de Dijon la seconde année (erreurs sur la chaine de saisie de données).

Les maïs ont été cultivés dans des conditions classiques similaires (avec irrigation à Lusignan et Toulouse). Les prélèvements ont débuté au plus tôt 20 j après la floraison, et se sont poursuivis au plus tard jusqu'à $82 \mathrm{j}$ après la floraison, à raison de 4-7 semaines de mesures selon les hybrides et les lieux.

Pour l'ensemble des 2 années, des 7 lieux et des 16 génotypes, ont ainsi été étudiés 237 maîs fourrage dont la teneur en matière sèche lors de la distribution aux animaux était comprise entre 18,3 et $53,1 \%$, avec une moyenne de $29,0 \%$. Les teneurs en grain de chacun de ces fourrages ont été estimées à partir d'un échantillon de 20 plantes.

Les digestibilités in vivo de ces fourrages ont été estimées par des moutons texels castrés, à l'entretien en cages à digestibilité dans les conditions standards. Les animaux sont affouragés avec le maïs grossièrement haché, distribué en plat unique à un niveau voisin de leurs besoins d'entretien ( $42 \mathrm{~g}$ de matière sèche par $\mathrm{kg}$ de poids métabolique), de sorte que l'ensemble du fourrage distribué soit consommé. La ration est équilibrée en azote et minéraux par l'apport de $30 \mathrm{~g}$ d'Urésil, contenant $15 \mathrm{~g}$ d'urée. Chacun de ces fourrages maïs est distribué 2 fois/j à 6 moutons pendant 5-6 j de mesures, après une période d'accoutumance de $10 \mathrm{j}$. Les teneurs en matière organique, azote et cellulose (méthodes Kjeldahl et Weende respectivement) ont été mesurées, pour le fourrage distribué et les fécès, à partir d'un échantillonnage représentatif des 6 moutons et des 5-6 j de mesures. Dans quelques cas, 1 ou 2 moutons ont été éliminés en raison de leur comportement anormal (quantités ingérées trop faibles en particulier). Les digestibilités ou coefficients d'utilisation digestive (CUD) de la matière organique, des matières azotées et de la cellulose brute sont estimés sur l'ensemble du lot de moutons pour chaque période de mesures. Les poids des moutons, homogènes sur chacun de lieux, se sont avérés va- riables sur les différents lieux, compris entre $52 \mathrm{~kg}$ à Dijon et $81 \mathrm{~kg}$ à La Jaillière.

Des échantillons représentatifs des maïs distribués aux moutons ont été, après broyage, répartis dans les différents laboratoires chargés des mesures in vitro.

Les teneurs en constituants chimiques classiques (matière organique, matières azotées totales, cellulose brute, lignine) et les teneurs en constituants pariétaux (NDF, ADF, respectivement Neutral et Acid Detergent Fiber, méthode van Soest) de chacun des 237 échantillons ont été mesurées par le laboratoire de l'ITCF de Boigneville.

Les digestibilités par solubilité enzymatique ont été réalisées par le laboratoire de l'ITCF (méthode Aufrère, 1982), par le laboratoire de I'INRA de Lusignan (méthode APC, Lila et al, 1986) et par le laboratoire de la station de Haute-Belgique, CRA de Gembloux (Libramont, méthode Limagrain-Ensitec, digestibilité de la matière sèche). La digestibilité avec du jus de rumen a été mesurée au laboratoire de I'INRA de Lusignan (méthode de Tilley et Terry, 1963). Les prédictions de la digestibilité par la méthode LibramontLimagrain et du CUD de la matière organique, après étalonnage dans le proche infrarouge, ont été faites par le laboratoire du CRA de Libramont en utilisant une régression au sens des carrés, partiels (Martens et Jensen, 1982).

Les résultats présentés ici correspondent à un regroupement des données des 2 années et des 7 lieux. Quatre études ont été conduites en parallèle, avec différents sous-ensembles des données, incluant ou non le génotype bm3, et en se limitant ou non aux fourrages ayant atteint un niveau de maturité au moins égal à ce qui est habituellement observé dans les conditions agricoles $(27,5 \%$ de matière sèche) :

- ensemble des 237 observations;

- ensemble de 229 observations, après élimination du génotype bm3;

- ensemble de 134 observations, en se limitant aux maïs dont la teneur en matière sèche du fourrage distribué est supérieure ou égale à $27,5 \%$;

- ensemble de 130 observations, en se limitant aux maïs dont la teneur en matière sèche du fourrage distribué $\geq 27,5 \%$, après élimination du génotype bm3. 
Les analyses de variance ont été réalisées sur ce dispositif non orthogonal et très déséquilibré avec le logiciel Modli (INRA, Biométrie), en prenant en compte :

- un effet année,

- un effet lieu,

- un effet génotype,

- une interaction génotype $x$ année,

- une interaction génotype $x$ lieu,

- un effet stade hiérarchisé aux combinaisons des lieux et des génotypes.

Par ailleurs, la mise en évidence d'un effet lieu pour un caractère tel que la digestibilité in vitro réalisée par un des laboratoires correspond essentiellement à une variabilité de comportement des hybrides en fonction du lieu de culture, alors que, par exemple, pour le CUD de la matière organique mesuré par les moutons standards, il y a confusion d'effet entre ce qui est induit par le milieu de culture comme dans le cas précédent, et une éventuelle variabilité ou différence de comportement des animaux ou des expérimentateurs sur les différents sites. Pour tenter de séparer ces 2 aspects, une étude limitée aux 2 hybrides les mieux répartis sur les différents lieux a été réalisée (hybrides 1 et 2 sur les lieux 1 à 6 ).

\section{RÉSULTATS ET DISCUSSION}

II y a, d'une manière générale, une assez grande dispersion des résultats, conduisant à de larges nuages de points comme cela est illustré par la figure 1 qui présente les relations entre la digestibilité in vitro méthode Libramont et respectivement le CUD de la matière organique et la teneur en ADF, pour l'ensemble des fourrages, ou en se limitant à ceux dont la teneur en matière sèche du distribué est au moins égale à $27,5 \%$. Une autre caractéristique générale de ces nuages est la position excentrée des fourrages du génotype bm3 pour les caractères de digestibilité. Ces nuages sont la résultante d'effets très différents (années, lieux, stade de récolte, génotype) et de leurs interactions. La compréhension des relations entre les caractères, l'évaluation de la pertinence de certaines mesures face à une problématique donnée et l'étude des possibilités éventuelles de prédiction de certaines qualités des génotypes suppose une structuration de la variabilité telle qu'elle peut, par exemple, être réalisée en analyse de variance.

\section{Variabilité phénotypique}

Les résultats des analyses de variance sont présentés dans les tableaux II a-d.

L'effet stade n'est significatif que pour des variables caractéristiques de la composition biochimique ou pariétale qui évoluent au cours de la maturation comme la teneur en amidon, en cellulose, en ADF, en lignine et à un moindre niveau pour la teneur en NDF (tableaux Ila et Ilb). II est généralement faible ou même non significatif pour les variables caractérisant une digestibilité, et juste significatif pour la valeur UFL (tableaux Ilc et IId). L'importance de l'effet année par rapport aux autres effets dépend de la variable considérée et, surtout, peut être très différente selon que l'on considère l'ensemble des observations ou les seuls fourrages dont la teneur en matière sèche est au moins égale à $27,5 \%$. Ainsi l'effet année est nettement plus important à teneur élevée en matière sèche pour la teneur en lignine et en cellulose brute, les CUD de la matière organique et de la cellulose, et donc, la valeur UFL. Ceci pourrait s'expliquer par des qualités de maturation différentes suivant les années, qui ne peuvent pas être mises en évidence quand on inclut dans l'étude des stades trop jeunes, mais qui ont des conséquences sur certaines caractéristiques de valeur alimentaire. La digestibilité par la méthode de Libramont a un comportement différent à ce niveau puisque c'est l'inverse qui est observé, avec un effet année significatif seulement quand on inclut les stades jeunes. Les effets lieux sont très généralement significatifs indiquant un effet moyen notable du milieu de culture sur les composants et sur la valeur alimentaire du maïs distribué. D'une manière générale, les interactions génotypes $x$ lieux ne sont pas significatives, sauf pour les CUD de la matère organique et de la cellulose mesurés par les moutons, et donc pour la valeur UFL. Si on se limite aux 2 génotypes les mieux représentés (hybrides 1 et 2, tableau III), les effets lieux sont du même ordre de grandeur pour les CUD de la matière organique ou de la cellulose que pour les 3 méthodes de digestibilité in vitro quand on inclut les stades précoces de récolte. En revanche, pour ces 2 hybrides, et pour les fourrages ayant une teneur en matière sèche au moins égale à $27,5 \%$, les effets lieux sont beau-

Fig 1. Relations entre la digestibilité in vitro estimée pour la méthode de Libramont et la teneur en fibres (ADF) ou le CUD de la matière organique mesurée par les moutons standards (les 7 (237 observations) et respectivement 4 points (134 observations) situés sur la partie droite des figures correspondent au génotype bm3). 

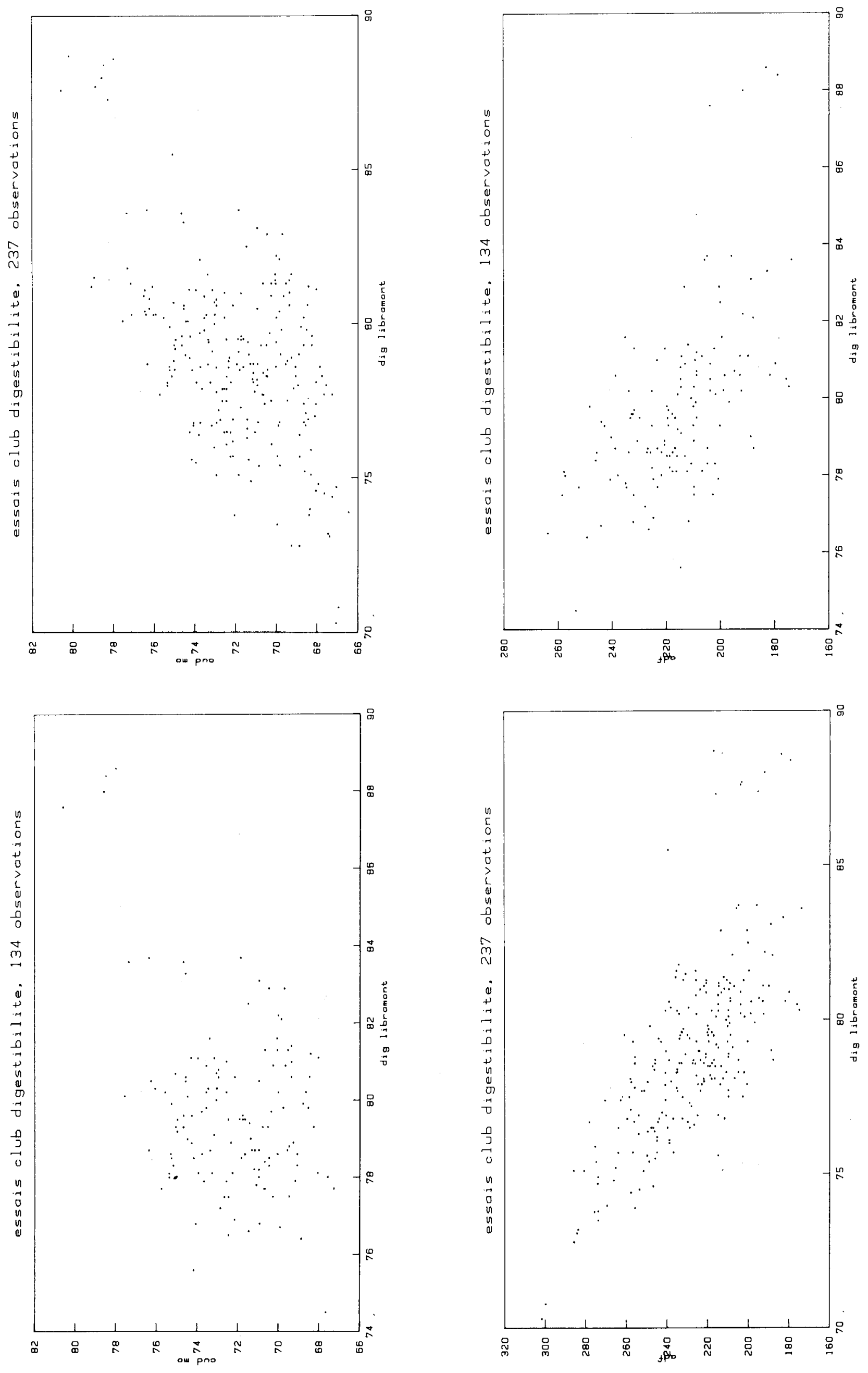
Tableau II. Analyses de variance pour les principaux caractères observés. Valeur et niveau de signification des variables $F$ de Fisher.

Ila. Caractéristiques agronomiques des fourrages distribués.

\begin{tabular}{|c|c|c|c|c|c|c|c|}
\hline \multirow{2}{*}{$\begin{array}{l}\text { Caractère } \\
\text { observé }\end{array}$} & \multicolumn{7}{|c|}{ Composante de la variance } \\
\hline & Année & Lieu & Génotype & Gén x lieu & Gén $x$ année & Gén/lieu/stade & $\sigma$ résiduel \\
\hline \multicolumn{8}{|c|}{ Matière sèche du distribué } \\
\hline 237 obs a $^{a}$ & $6,6^{*}$ & $29,7^{\star \star \star}$ & $3,3^{\star \star \star}$ & $3,4^{\star \star}$ & $2,5^{\star *}$ & $4,0^{* * *}$ & 3,2 \\
\hline $\mathrm{ddl}$ & 1 & 6 & 15 & 5 & 13 & 118 & 78 \\
\hline 229 obs $^{b}$ & $6,1^{*}$ & $28,6^{* \star *}$ & $3,4^{\star \star \star}$ & 3,3 * & 2,6 *夫 & $3,8^{\star \star \star}$ & 3,3 \\
\hline $\mathrm{ddl}$ & 1 & 6 & 14 & 5 & 12 & 115 & 75 \\
\hline 134 obs $^{c}$ & $36,4^{* \star *}$ & 6,6 *** & $1,3 \mathrm{~ns}$ & $1,2 \mathrm{~ns}$ & $1,0 \mathrm{~ns}$ & $2,1 *$ & 3,4 \\
\hline $\mathrm{ddl}$ & 1 & 6 & 15 & 5 & 12 & 71 & 23 \\
\hline 130 obs $^{d}$ & $31,8^{\star \star \star}$ & $6,3^{* * *}$ & $1,4 \mathrm{~ns}$ & $1,1 \mathrm{~ns}$ & $1,0 \mathrm{~ns}$ & $2,0^{*}$ & 3,5 \\
\hline ddl & 1 & 6 & 14 & 5 & 11 & 70 & 22 \\
\hline \multicolumn{8}{|c|}{ Teneur en grains } \\
\hline 237 obs $^{a}$ & $15,2^{\star \star \star}$ & $14,5^{* * *}$ & $4,3^{* * *}$ & $6,1^{* \star *}$ & $3,9^{\star \star \star}$ & $7,1^{* \star \star}$ & 4,7 \\
\hline 229 obs b $^{b}$ & $11,3^{\star \star}$ & 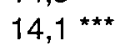 & $4,5^{* * *}$ & $5,9^{\star \star \star}$ & $3,7^{* * *}$ & $6,9^{\star \star \star}$ & 4,8 \\
\hline 134 obs $^{c}$ & $17,2^{* \star *}$ & $7,1^{\star \star \star}$ & 3,8 ** & 3,4 * & $2,0 \mathrm{~ns}$ & $3,8^{\star \star \star *}$ & 3,1 \\
\hline 130 obs $^{d}$ & $16,5^{\star \star \star \star}$ & $7,0^{\star \star \star}$ & $3,9^{* *}$ & 3,3 * & $2,1 \mathrm{~ns}$ & $3,7^{\star \star \star}$ & 3,1 \\
\hline \multicolumn{8}{|c|}{ Amidon EWERS } \\
\hline 237 obs $^{a}$ & $20,0^{* * *}$ & $8,8^{* \star \star}$ & $3,1^{* *}$ & $1,4 \mathrm{~ns}$ & 1,9 * & $4,7^{\star \star \star}$ & 4,2 \\
\hline 229 obs $^{b}$ & 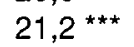 & $9,2^{\star \star \star}$ & $3,4^{* * *}$ & $1,5 \mathrm{~ns}$ & $2,1^{*}$ & $4,8^{\star \star \star}$ & 4,1 \\
\hline 134 obs $^{c}$ & $68,7^{\star \star \star}$ & $10,8^{\star \star \star}$ & $4,3^{* * *}$ & $2,2 \mathrm{~ns}$ & $4,7^{\star \star}$ & $3,4^{\star \star}$ & 2,2 \\
\hline 130 obs $^{d}$ & $73,8^{\star \star \star *}$ & $10,3^{\star \star \star}$ & $4,3^{\star \star \star}$ & 2,1 * & $4,0^{* *}$ & $3,2^{\star *}$ & 2,2 \\
\hline
\end{tabular}

a 237 observations; ${ }^{b} 229$ observations bm3 exclu; ${ }^{c} 134$ observations, matière sèche $\geq 27,5 ;{ }^{d} 130$ observations, matière sèche $\geq$ $27,5, \mathrm{bm}^{3}$ exclu. ${ }^{*}$ Résultat significatif au seuil de $5 \%$; ${ }^{\star \star}$ résultat significatif au seuil de $1 \% ;{ }^{* \star *}$ résultat significatif au seuil de $1 \%$.

coup plus importants pour les caractères de digestibilité mesurés par les animaux, que pour ceux qui sont mesurés in vitro, les 2 génotypes ayant dans ce cas des teneurs moyennes en matière sèche et en grain semblables (respectivement voisines de 32 et $45 \%$ ). Ces observations rejoignent celles notées sur l'ensemble des hybrides. II pourrait donc exister un effet sur les CUD lié au site expérimental utilisé, au moins pour ces fourrages ayant atteint un certain niveau de maturité et de teneur en grain. Outre l'environnement en général, cet effet peut éventuellement être relié aux différences de poids des animaux sur chacun des lieux. Les interactions génotypes $x$ année sont généralement significatives pour les caractéristiques analytiques mesurées et pour les caractères mesurés par les moutons. Elles le sont également pour les digestibilités mesurées par solubilité enzymatique quand on inclut les échantillons à faible teneur en matière sèche. En revanche, pour les fourrages dont la teneur en matière sèche est au moins égale à $27,5 \%$, l'interaction génotype $x$ année est non significative pour la méthode de Libramont, très faible pour la méthode de Lusignan, et faible pour la méthode utilisée par I'ITCF. Pour l'ensemble de ces effets ou interactions, il n'existe pas de différence notable de niveau de chacun d'eux selon que l'on exclut ou non le génotype bm3 de l'étude. À l'inverse, les effets génotypiques peuvent différer très nettement en présence ou non du génotype bm3. Ils ne sont significatifs pour la teneur en lignine et le CUD de la matière organique que si le génotype bm3 est inclus. Les valeurs des tests de Fisher sont $2-3$ fois plus faibles pour le CUD de la cellulose et les solubilités enzymatiques en absence du génotype bm3, à niveau d'erreur résiduelle égale. Par ailleurs, les écarts types résiduels des CUD de la matière organique, de la cellulose, et 
Ilb. Caractéristiques liées à la qualité des parois des fourrages distribués.

\begin{tabular}{|c|c|c|c|c|c|c|c|}
\hline \multirow{2}{*}{$\begin{array}{l}\text { Caractère } \\
\text { observé }\end{array}$} & \multicolumn{7}{|c|}{ Composante de la variance } \\
\hline & Année & Lieu & Génotype & Gén x lieu & Gén $x$ année & Gén/lieu/stade & $\sigma$ résiduel \\
\hline \multicolumn{8}{|c|}{ Cellulose brute } \\
\hline 237 obs $^{a}$ & $2,4 \mathrm{~ns}$ & $10,5^{\star \star \star}$ & $5,9^{* \star \star}$ & $0,3 \mathrm{~ns}$ & $3,1^{* *}$ & $2,2^{* * *}$ & 1,4 \\
\hline 229 obs $^{b}$ & $2,8 \mathrm{~ns}$ & $10,5^{* * *}$ & $5,6^{\star \star \star}$ & $0,3 \mathrm{~ns}$ & $3,4^{\star \star *}$ & $2,2^{\star \star \star}$ & 1,4 \\
\hline 134 obs $^{c}$ & $12,9^{\star \star}$ & 23,9 * & $6,1^{\star \star \star}$ & $0,5 \mathrm{~ns}$ & 3,9 ** & $1,0 \mathrm{~ns}$ & 1,0 \\
\hline 130 obs $^{d}$ & $15,0^{* *}$ & $23,0^{* * *}$ & $5,5^{* * *}$ & $0,5 \mathrm{~ns}$ & $3,6^{* *}$ & $1,0 \mathrm{~ns}$ & 1,0 \\
\hline \multicolumn{8}{|l|}{ NDF } \\
\hline 237 obs $^{a}$ & $1,9 \mathrm{~ns}$ & $8,4^{\star \star \star}$ & $3,0^{* \star *}$ & $0,9 \mathrm{~ns}$ & $2,1^{*}$ & $1,9^{\star *}$ & 2,5 \\
\hline 229 obs $^{b}$ & $1,5 \mathrm{~ns}$ & $8,3^{* * *}$ & $3,2^{* * *}$ & $0,9 \mathrm{~ns}$ & $2,2^{*}$ & $1,8^{\star \star}$ & 2,5 \\
\hline 134 obs $^{c}$ & $2,0 \mathrm{~ns}$ & $12,0^{* \star \star}$ & $3,0^{* *}$ & $0,5 \mathrm{~ns}$ & $3,2^{* *}$ & $1,1 \mathrm{~ns}$ & 2,2 \\
\hline 130 obs $^{d}$ & $1,3 \mathrm{~ns}$ & $11,4^{* * *}$ & $3,0^{*}$ & $0,5 \mathrm{~ns}$ & $3,2^{\star \star}$ & $1,0 \mathrm{~ns}$ & 2,2 \\
\hline \multicolumn{8}{|l|}{$A D F$} \\
\hline 237 obs $^{a}$ & 5,4 * & $8,9^{* \star \star}$ & $5,9^{\star \star \star}$ & $0,9 \mathrm{~ns}$ & $3,4^{* \star \star}$ & $2,9^{\star * *}$ & 1,5 \\
\hline 229 obs b $^{b}$ & $6,2^{*}$ & $8,8^{* * *}$ & $5,2^{\star \star \star}$ & $0,9 \mathrm{~ns}$ & $3,6^{* * *}$ & $2,9^{* * *}$ & 1,5 \\
\hline 134 obs $^{c}$ & $2,0 \mathrm{~ns}$ & $17,9^{* \star *}$ & $5,2^{\star \star *}$ & $1,0 \mathrm{~ns}$ & 3,6 ** & $1,2 \mathrm{~ns}$ & 1,1 \\
\hline 130 obs $^{d}$ & $1,3 \mathrm{~ns}$ & $17,1^{\star \star \star}$ & $4,4^{\star \star}$ & $1,0 \mathrm{~ns}$ & $3,4^{* *}$ & $1,2 \mathrm{~ns}$ & 1,1 \\
\hline \multicolumn{8}{|l|}{ Lignine } \\
\hline 237 obs $^{a}$ & 0,0 ns & $8,4^{* * *}$ & $12,7^{\star \star \star}$ & $3,7^{* \star}$ & $3,8^{* \star \star}$ & $2,4^{\star \star *}$ & 2,5 \\
\hline 229 obs $^{b}$ & $0,0 \mathrm{~ns}$ & $8,4^{\star * *}$ & 3,1 ** & $3,7^{\star \star}$ & $4,1^{* \star \star}$ & $2,4^{\star \star \star}$ & 2,5 \\
\hline 134 obs $^{c}$ & $11,5^{\star \star}$ & 3,5 * & $8,0^{* * *}$ & $1,4 \mathrm{~ns}$ & $3,8^{* *}$ & $1,1 \mathrm{~ns}$ & 0,2 \\
\hline 130 obs $^{d}$ & 13,5 ** & $1,7^{*}$ & 2,6 * & $1,3 \mathrm{~ns}$ & $4,0^{* *}$ & $1,0 \mathrm{~ns}$ & 0,2 \\
\hline
\end{tabular}

a 237 observations; ${ }^{b} 229$ observations bm3 exclu; ${ }^{c} 134$ observations, matière sèche $\geq 27,5 ;{ }^{d} 130$ observations, matière sèche $\geq$ $27,5, \mathrm{bm} 3$ exclu. " Résultat significatif au seuil de $5 \%$; "* résultat significatif au seuil de $1 \%$; *** résultat significatif au seuil de $1 \%$.

ceux des digestibilités in vitro sont plus faibles pour les fourrages récoltés à une teneur en matière sèche supérieure ou égale à $27,5 \%$. Puisque les valeurs des tests de Fisher pour ces 2 CUD, comme celles des solubilités enzymatiques selon les méthodes de Libramont et Lusignan, sont supérieures sur le sous-ensemble des fourrages les plus riches en matière sèche, il existerait donc, au niveau normal de maturité, une variabilité phénotypique entre génotypes supérieure à celle existant à des niveaux plus faibles. Enfin, la solubilité enzymatique de Lusignan semble plus discriminante quand elle est appliquée aux seuls fourrages dont la teneur en matière sèche est d'au moins $27,5 \%$, alors que c'est le contraire pour la méthode appliquée par I'ITCF, et que la méthode de Libramont conserve la même qualité discriminante quel que soit le niveau de maturité des plantes, avec toujours l'écart type résiduel le plus faible. Les digestibilités in vitro prédites par réflectance dans le proche infrarouge ont un comportement très semblable à celui des ana- lyse de référence correspondantes, avec toutefois une précision supérieure à la référence pour les échantillons à un niveau de maturité normal, et donc un meilleur pouvoir discriminant pour les effets lieux et génotypes en particulier (tableau IId). II en est de même pour le CUD de la matière organique prédit en NIRS, avec en particulier un effet génotype qui est significatif pour le sous-ensemble des fourrages les plus avancés en maturité, fourrages bm3 exclu, alors que ce n'est pas le cas pour les valeurs directement mesurées par les animaux (tableau IIc). Les prédictions faites après analyses de spectres intègrent donc une structuration de la variation, et conduisent à des valeurs prédites plus "précises" que les valeurs de référence. Pour le CUD de la matière organique, elles conduisent de plus à la suppression de l'interaction génotype $x$ lieu. La méthode de Tilley et Terry, dans les conditions d'utilisation employées ici, à savoir après adaptation à l'analyse d'un grand nombre d'échantillons par unité de temps, et peu 
Ilc. Caractéristiques mesurées par les moutons sur les fourrages distribués.

\begin{tabular}{|c|c|c|c|c|c|c|c|}
\hline \multirow{2}{*}{$\begin{array}{l}\text { Caractère } \\
\text { observé }\end{array}$} & \multicolumn{7}{|c|}{ Composante de la variance } \\
\hline & Année & Lieu & Génotype & Gén x lieu & Gén x année & Gén/lieu/stade & $\sigma$ résiduel \\
\hline \multicolumn{8}{|c|}{ CUD matière org } \\
\hline 237 obs $^{a}$ & $30,9 * \star \star$ & $12,6^{\star * *}$ & $7,0^{* \star *}$ & $4,2^{* *}$ & $5,4^{* * *}$ & $1,4 \mathrm{~ns}$ & 1,7 \\
\hline 229 obs $^{b}$ & $28,1^{\star \star \star}$ & $12,3^{* \star \star}$ & $1,8 \mathrm{~ns}$ & $4,1 * *$ & $5,7^{* * *}$ & $1,4 \mathrm{~ns}$ & 1,7 \\
\hline 134 obs $^{c}$ & $156,6^{\star \star \star *}$ & $28,0^{* * *}$ & $8,2^{\star * \star}$ & $6,6^{\star \star *}$ & $2,1 \mathrm{~ns}$ & 1,9 * & 1,0 \\
\hline 130 obs $^{d}$ & $159,6^{\star \star \star}$ & $28,5^{\star \star \star}$ & $2,0 \mathrm{~ns}$ & $6,7^{\star \star \star}$ & $2,3^{*}$ & $2,0^{*}$ & 1,0 \\
\hline \multicolumn{8}{|c|}{ CUD cellulose } \\
\hline 237 obs $^{a}$ & $13,5^{* * *}$ & $5,7^{\star \star \star}$ & $10,2^{\star \star \star}$ & $4,5^{\star *}$ & $4,9^{\star \star \star}$ & $0,7 \mathrm{~ns}$ & 3,6 \\
\hline 229 obs $^{b}$ & 10,1 ** & $5,7^{\star \star \star}$ & $5,1^{* \star *}$ & $4,5 * \star$ & $4,9^{\star \star \star}$ & $0,7 \mathrm{~ns}$ & 3,7 \\
\hline 134 obs $^{c}$ & $80,5^{\star \star \star}$ & $11,1 \star \star \star$ & $11,2^{* \star \star}$ & $5,6^{\star \star}$ & $3,9^{\star *}$ & 1,9 * & 2,3 \\
\hline 130 obs $^{d}$ & $77,1^{\star \star \star \star}$ & $11,6^{\star \star *}$ & $6,2^{\star \star \star \star}$ & $5,9^{\star \star}$ & $4,2^{\star \star}$ & $2,0^{*}$ & 2,3 \\
\hline \multicolumn{8}{|l|}{$U F L$} \\
\hline 237 obs $^{a}$ & $44,8^{\star \star \star}$ & $12,8^{\star \star \star}$ & $6,4^{\star \star \star}$ & $3,8^{\star \star}$ & $4,1^{\star \star \star}$ & $1,9^{* \star}$ & 0,03 \\
\hline 229 obs b $^{b}$ & $43,7^{* * *}$ & $12,4^{\star \star \star}$ & $1,9^{*}$ & 3,6 ** & $4,3^{* * *}$ & 1,9 ** & 0,03 \\
\hline 134 obs $^{c}$ & $160,1 * \star \star$ & $29,9^{\star \star \star}$ & $7,7^{\star \star \star}$ & $6,2^{* *}$ & $2,4^{*}$ & $2,0^{*}$ & 0,00 \\
\hline 130 obs $^{d}$ & $163,4^{\star \star \star}$ & $29,7^{\star \star *}$ & $2,0 \mathrm{~ns}$ & $6,1^{* *}$ & 2,4 * & $2,1^{* *}$ & 0,00 \\
\hline \multicolumn{8}{|c|}{ CUD mat org prédit } \\
\hline 237 obs $^{a}$ & $26,6^{\star \star \star}$ & $5,9^{\star \star \star}$ & $5,8^{* * *}$ & $0,4 \mathrm{~ns}$ & $4,2^{\star \star \star}$ & $0,6 \mathrm{~ns}$ & 1,9 \\
\hline 229 obs $^{b}$ & $14,4^{* \star *}$ & $5,7^{\star \star \star}$ & $1,4 \mathrm{~ns}$ & $0,4 \mathrm{~ns}$ & $4,2^{\star \star *}$ & $0,6 \mathrm{~ns}$ & 1,9 \\
\hline 134 obs $^{c}$ & $181,9^{\star \star \star}$ & $22,8^{\star \star \star}$ & $11,7^{\star \star \star}$ & $1,4 \mathrm{~ns}$ & $2,1 \mathrm{~ns}$ & $0,9 \mathrm{~ns}$ & 0,9 \\
\hline 130 obs $^{d}$ & $167,3^{* * *}$ & $21,9 * * *$ & $3,7^{\star *}$ & $1,3 \mathrm{~ns}$ & $2,2 \mathrm{~ns}$ & $0,9 \mathrm{~ns}$ & 0,9 \\
\hline
\end{tabular}

a 237 obsẹvations; ${ }^{b} 229$ observations bm3 exclu; ${ }^{c} 134$ observations, matière sèche $\geq 27,5 ;{ }^{d} 130$ observations, matière sèche $\geq$ $27,5, \mathrm{bm} 3$ exclu. * Résultat significatif au seuil de $5 \%$; ${ }^{* *}$ résultat significatif au seuil de $1 \%$; ${ }^{* * *}$ résultat significatif au seuil de $1 \%$.

de répétitions analytiques ne semble pas présenter d'intérêt pour le tri de génotypes puisqu'elle ne permet pas de révéler une variabilité en raison d'un écart type résiduel élevé (tableau IId).

\section{Estimation des effets moyens}

Les effets principaux ne sont pas estimables en présence d'interactions significatives entre les facteurs et de l'effet stade hiérarchisé aux autres facteurs. Toutefois, cet effet stade est faible ou non significatif pour la plupart des variables estimant la valeur alimentaire, et il en est de même de l'interaction génotype $x$.lieu pour les fourrages dont la teneur en matière sèche est au moins égale à $27,5 \%$; une estimation des effets moyens a donc été envisagée avec un modèle restreint aux effets principaux et à l'interaction génotype $x$ année. Dans ces conditions, seule la valeur moyenne de 13-16 génotypes est estimable. Nous avons alors considéré que l'interaction génotype $x$ année est suffisamment faible et négligeable par rapport aux effets principaux pour estimer des valeurs moyennes avec un modèle additif. L'étude de ces valeurs moyennes (tableaux IVa, IVb) illustre les résultats mis en évidence par l'analyse de variance. L'hybride $\mathrm{bm}^{3}$, qui ne se différencie pas ou très peu des hybrides normaux pour la teneur en grain, cellulose, NDF et même en ADF a en revanche une teneur en lignine réduite de presque moitié et une digestibilité de la matière organique et surtout de la cellulose nettement plus élevées. Ces caractéristiques du matériel bm3 sont également bien mises en évidence par les différentes méthodes in vitro. On retrouve ici l'importance des maillages lignines $x$ celluloses dans la digestibilité d'un fourrage puisque, à teneur en cellulose et 
Ild. Différentes digestibilités estimées des fourrages distribués.

\begin{tabular}{|c|c|c|c|c|c|c|c|}
\hline \multirow{2}{*}{$\begin{array}{l}\text { Caractère } \\
\text { observé }\end{array}$} & \multicolumn{7}{|c|}{ Composante de la variance } \\
\hline & Année & Lieu & Génotype & Gén x lieu & Gén x année & Gén/lieu/stade & $\sigma$ résiduel \\
\hline \multicolumn{8}{|c|}{ Digestibilité ITCF } \\
\hline 237 obs $^{a}$ & $1,6 \mathrm{~ns}$ & $13,0^{* * *}$ & $14,1^{\star \star *}$ & $0,2 \mathrm{~ns}$ & $5,7^{\star \star \star}$ & 1,9 ** & 1,7 \\
\hline 229 obs $^{b}$ & $0,5 \mathrm{~ns}$ & $12,6^{\star \star \star}$ & $5,1^{\star \star \star}$ & $0,2 \mathrm{~ns}$ & $5,4^{\star \star \star}$ & $1,9^{\star *}$ & 1,7 \\
\hline 134 obs $^{c}$ & $0,3 \mathrm{~ns}$ & $15,6^{\star * \star}$ & $10,1^{\star \star \star}$ & $0,8 \mathrm{~ns}$ & $5,3^{* \star \star}$ & $1,1 \mathrm{~ns}$ & 1,4 \\
\hline 130 obs $^{d}$ & $1,5 \mathrm{~ns}$ & $14,6^{* * *}$ & $3,8^{* *}$ & $0,8 \mathrm{~ns}$ & $4,4^{* *}$ & $1,0 \mathrm{~ns}$ & 1,4 \\
\hline \multicolumn{8}{|c|}{ Digestibilité Libramont } \\
\hline 237 obs $^{a}$ & $7,9^{\star \star}$ & $12,6^{\star \star \star}$ & $16,2^{\star \star \star}$ & $0,6 \mathrm{~ns}$ & $3,7^{\star \star \star}$ & 1,5 * & 1,5 \\
\hline 229 obs $^{b}$ & $8,4^{\star \star}$ & $12,2^{* * *}$ & $5,0^{\star * *}$ & $0,6 \mathrm{~ns}$ & $3,9 * \star *$ & $1,5^{*}$ & 1,6 \\
\hline 134 obs $^{c}$ & $2,8 \mathrm{~ns}$ & $15,7^{\star \star \star}$ & $16,6^{\star \star \star}$ & $2,3 \mathrm{~ns}$ & $1,9 \mathrm{~ns}$ & $1,2 \mathrm{~ns}$ & 1,0 \\
\hline 130 obs $^{d}$ & $2,4 \mathrm{~ns}$ & $15,1^{\star \star *}$ & $6,2^{* \star \star}$ & $2,2 \mathrm{~ns}$ & $2,0 \mathrm{~ns}$ & $1,1 \mathrm{~ns}$ & 1,0 \\
\hline \multicolumn{8}{|l|}{ Digestibilité } \\
\hline \multicolumn{8}{|c|}{ APC Lusignan } \\
\hline 237 obs $^{a}$ & $19,3^{* \star *}$ & 2,0 ns & $12,2^{* * *}$ & $0,2 \mathrm{~ns}$ & $2,8 * \star$ & $1,4 \mathrm{~ns}$ & 2,1 \\
\hline 229 obs b & $19,1 \star \star \star$ & $2,0 \mathrm{~ns}$ & $3,7^{\star \star \star}$ & $0,2 \mathrm{~ns}$ & 2,9 * & $1,4 \mathrm{~ns}$ & 2,2 \\
\hline 134 obs $^{c}$ & $11,2^{* \star}$ & $5,7^{\star \star}$ & $16,9^{\star \star \star}$ & $0,8 \mathrm{~ns}$ & $3,0^{*}$ & $1,9^{*}$ & 1,3 \\
\hline 130 obs $^{d}$ & $9,2^{* *}$ & $5,5^{\star *}$ & $6,7^{* * *}$ & $0,7 \mathrm{~ns}$ & $3,0^{*}$ & $1,9^{*}$ & 1,3 \\
\hline \multicolumn{8}{|l|}{ Tilley Terry } \\
\hline \multicolumn{8}{|l|}{ Lusignan } \\
\hline 237 obs $^{a}$ & 1,4 ns & $6,3^{\star \star \star}$ & $3,2^{\star \star \star}$ & $0,9 \mathrm{~ns}$ & $3,2^{* * *}$ & $0,6 \mathrm{~ns}$ & 3,8 \\
\hline 229 obs $^{b}$ & $1,5 \mathrm{~ns}$ & $6,1^{\star \star \star}$ & $1,5 \mathrm{~ns}$ & $0,9 \mathrm{~ns}$ & $3,4^{\star \star \star}$ & $0,6 \mathrm{~ns}$ & 3,8 \\
\hline 134 obs $^{c}$ & 2,2 ns & $7,5^{\star \star \star}$ & 1,9 ns & $1,1 \mathrm{~ns}$ & $2,5^{*}$ & $0,8 \mathrm{~ns}$ & 3,2 \\
\hline 130 obs $^{d}$ & $1,9 \mathrm{~ns}$ & $7,2^{\star \star \star}$ & $1,4 \mathrm{~ns}$ & $1,1 \mathrm{~ns}$ & $2,7^{*}$ & $0,7 \mathrm{~ns}$ & 3,3 \\
\hline \multicolumn{8}{|c|}{ Digestibilité prédite } \\
\hline \multicolumn{8}{|c|}{ NIRS Libramont } \\
\hline 237 obs $^{a}$ & $7,4^{\star \star}$ & $12,8^{\star \star \star}$ & $16,8^{\star \star \star}$ & $0,3 \mathrm{~ns}$ & $3,3^{\star \star *}$ & $1,4 \mathrm{~ns}$ & 1,5 \\
\hline 229 obs $^{b}$ & $6,9^{*}$ & $12,4^{\star \star \star}$ & $4,8^{\star \star \star}$ & $0,3 \mathrm{~ns}$ & $3,5^{\star \star \star}$ & $1,4 \mathrm{~ns}$ & 1,5 \\
\hline 134 obs $^{c}$ & $3,8 \mathrm{~ns}$ & $23,6^{\star \star \star}$ & $25,8^{* \star *}$ & $0,6 \mathrm{~ns}$ & $1,7 \mathrm{~ns}$ & 1,4 ns & 0,8 \\
\hline 130 obs $^{d}$ & $2,5 \mathrm{~ns}$ & $22,5^{\star \star \star}$ & $9,3^{\star \star \star}$ & $0,5 \mathrm{~ns}$ & $1,6 \mathrm{~ns}$ & $1,4 \mathrm{~ns}$ & 0,8 \\
\hline
\end{tabular}

a 237 observations; ${ }^{b} 229$ observations bm3 exclu; ${ }^{c} 134$ observations, matière sèche $\geq 27,5 ;{ }^{d} 130$ observations, matière sèche $\geq$ 27,5 , bm3 exclu. * Résultat significatif au seuil de $5 \%$; ** résultat significatif au seuil de $1 \%$; ${ }^{* * *}$ résultat significatif au seuil de $1 \%$.

en grain semblables à celles des autres hybrides, la forte diminution de la teneur en lignine du génotype bm3 conduit à une digestibilité de la cellulose nettement plus élevée. La variabilité phénotypique du matériel étudié peut paraître relativement faible, en particulier quand on exclut l'hybride bm3, avec des écarts entre le minimum et le maximum valant respectivement $6,9,4,9$ et 6,1 points pour chacune des 3 méthodes de digestibilité in vitro, valeurs à pondérer par leurs précisions respectives. Mais cette variation est représentative de ce que l'on observe couramment entre les hybrides des catalogues européens, et semble même un peu supérieure à celle observée par Deinum et Struik (1985), sur une collection d'hybrides du catalogue néerlandais. Les 3 solubilités enzymatiques donnent des résultats différents, avec, en particulier, une valeur moyenne plus faible pour la méthode utilisée par l'ITCF, mais classent de façon similaire les génotypes si on les répartit en 2 ou 3 grands groupes de comportement (fig 2). En effet, l'observation des valeurs moyennes, dans le sousensemble représentatif du niveau de maturité au stade de récolte pour l'ensilage, montre que les 3 solubilités enzymatiques conduisent au même résultat pour le tri des hybrides dont la digestibilité est la plus élevée, le 2 et le 12, outre le 17 
Tableau III. Digestibilités in vivo et in vitro, ADF, pour les hybrides 1 et 2 . Valeur et niveau de signification des variables $F$ de Fisher. a : Ensemble des 65 observations. $\mathbf{b}$ : Ensemble des 39 observations dont la teneur en matière sèche est $\geq 27,5 \%$.

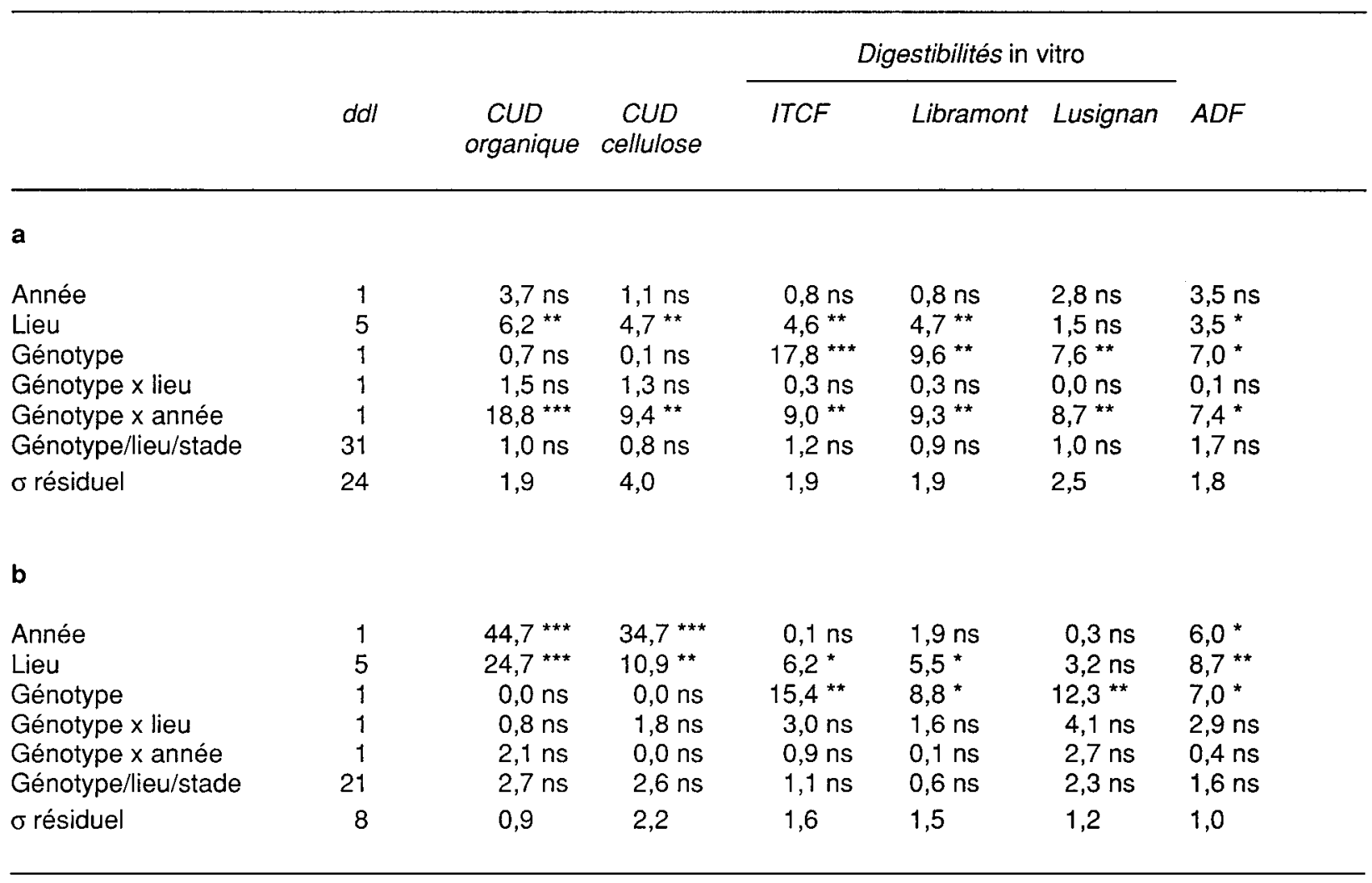

(bm3), ou la plus faible, le 14 , le 3 et le 6 . La réponse des hybrides intermédiaires semble varier en fonction de la méthode enzymatique utilisée. Mais les classements sont en fait difficiles à réaliser pour des hybrides dont la valeur est proche, avec des intervalles de confiance de l'ordre de grandeur des différences observées. Parallèlement, avec un écart entre le minimum et le maximum de 3,2 points, hormis le génotype bm3, la variabilité mise en évidence par les moutons pour le CUD de la matière organique est faible, et même non significative. Toutefois les hybrides 2 et 12 sont parmi ceux dont la valeur de CUD de la matière organique estimée par les moutons standards est la plus élevée $(73,8$ et 73,5$)$, et les hybrides 3 et 14 sont parmi ceux dont la valeur de CUD est faible $(71,9$ et 71,0$)$, l'hybride 6 se classant de manière intermédiaire. Enfin, la variabilité mise en évidence pour la digestibilité ne semble pas directement liée à la précocité, même si certains résultats obtenus sur moutons montrent une tendance à une diminution de la digestibilité lorsque la tardiveté augmente (Bar- rière et al, 1988). Les 2 hybrides les plus tardifs (6 et 14) observés ici se classent effectivement parmi ceux dont la digestibilité est faible. En revanche, l'hybride 5, demi-tardif, a une digestibilité de type intermédiaire. Les hybrides dont la digestibilité semble la plus élevée sont des types précoces ou demi-précoces, mais l'hybride 3 , dont la digestibilité est faible, est aussi un type demi-précoce.

\section{Corrélations}

Les corrélations sont dues à l'ensemble des effets du modèle complet d'analyse de variance.

Les 3 méthodes de mesures in vitro de la digestibilité paraissent bien corrélées entre-elles quand on prend en compte l'ensemble des fourrages testés (tableau $\mathrm{Va}$ ). En fait ces liaisons apparaissent, pour cet ensemble, surestimées de 0,07 à 0,15 points par la présence du génotype $\mathrm{bm}_{3}$, dont les valeurs de digestibilité sont plus

Fig 2. Relations entre les moyennes phénotypiques des 16 génotypes pour la digestibilité in vitro méthode Libramont, et respectivement la digestibilité in vitro méthode APC, le CUD de la matière organique, la digestibilité in vitro réalisée par l'ITCF. 
essais club digestibilite. 16 genotypes

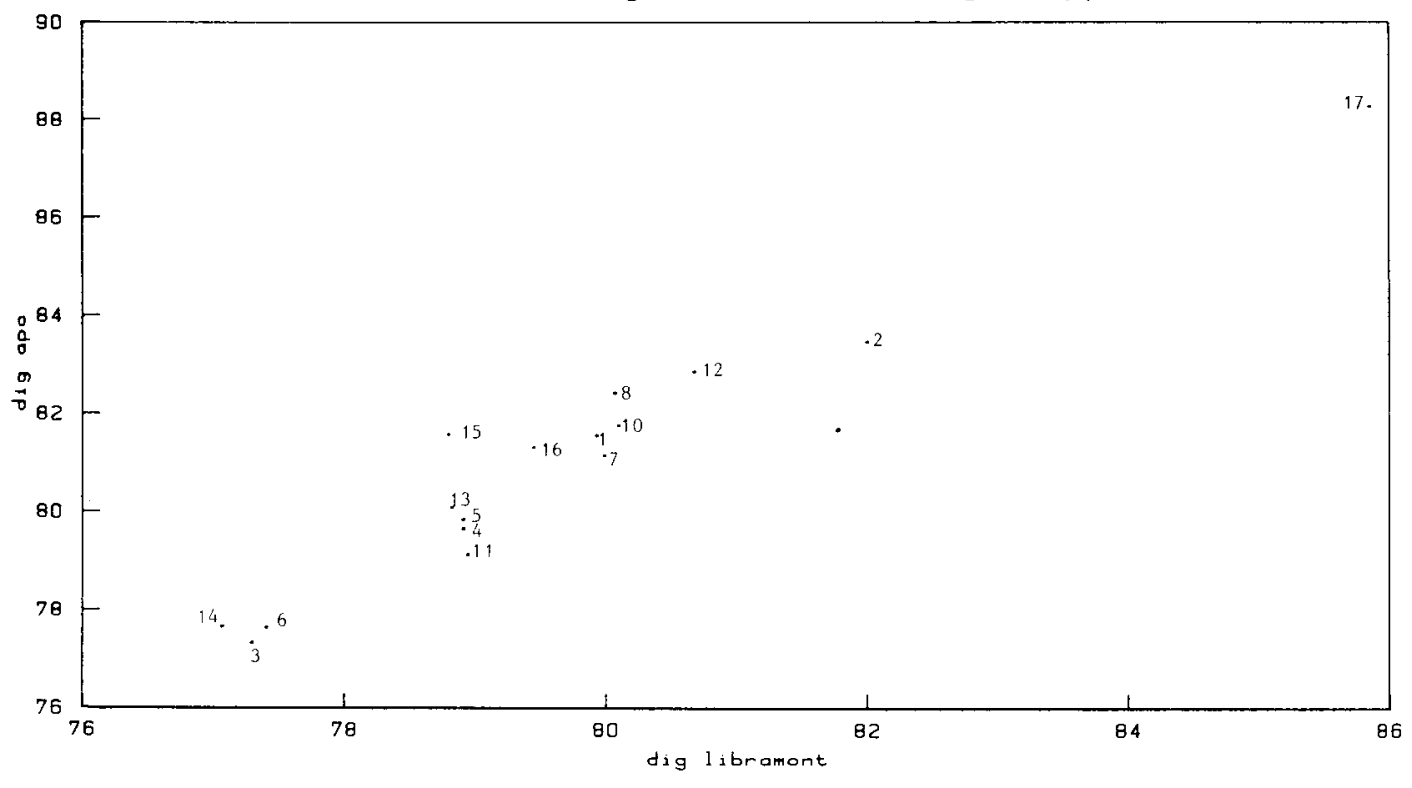

essais club digestibilite. 16 genotypes
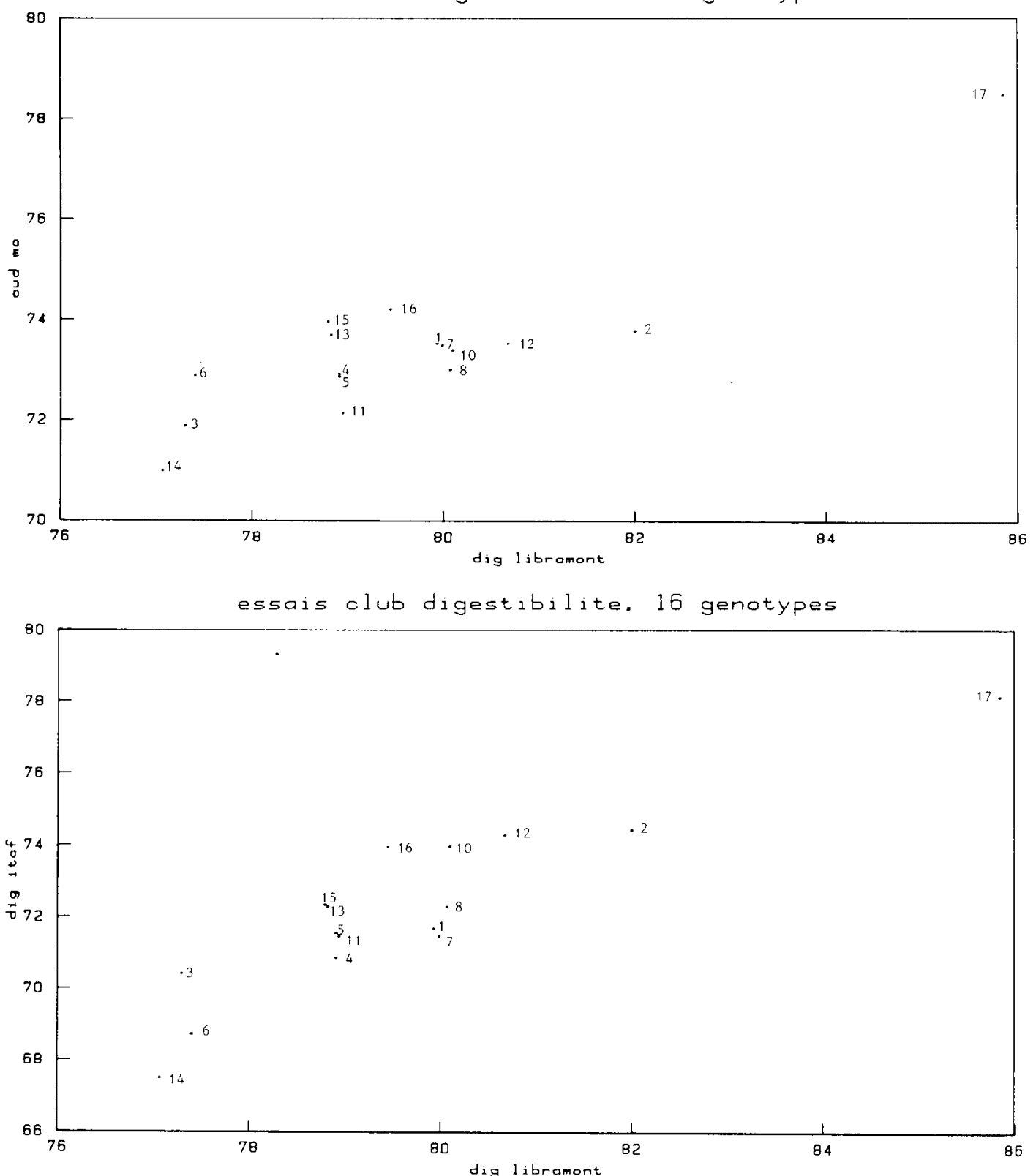


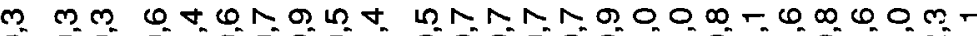

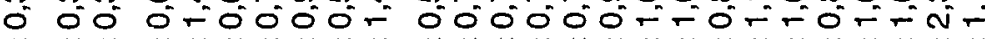
$+1+1+1+1+1+1+1+1+1+1+1+1+1+1+1+1+1+1+1+1+1+1+1+1+1+1$

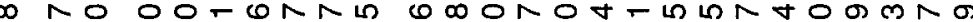

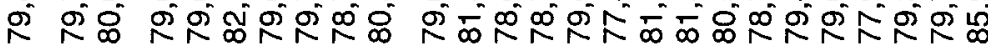

L o 0 $+1+1+1+1+1+1+1+1+1+1+111+1+1+1+1+1+1+1+1+1+1+1+1+1+1$

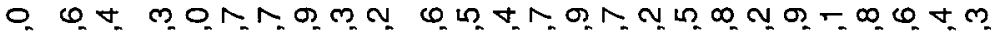

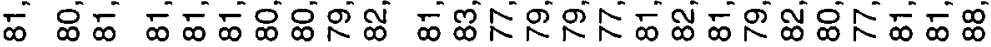

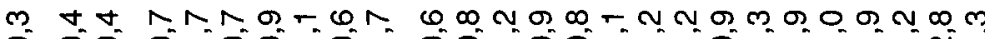

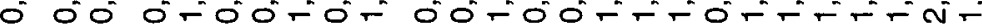
$+1+1+1+1+1+1+1+1+1+1+1+1+1+1+1+1+1+1+1+1+1+1+1+1+1+1$

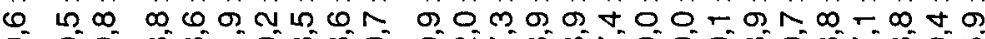

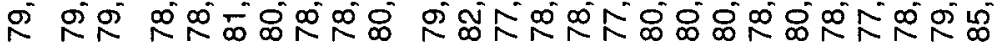

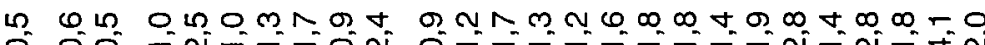
$000+1+1+1+1+1+1+1+1+1+-1+1+1+1+1+1+1+1$

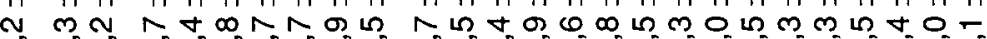

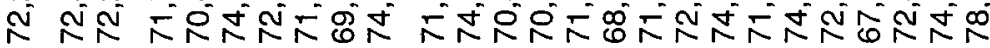

9. ㄱ. OOOLMNG

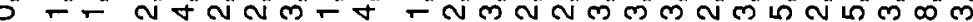
$+1+1+1+1+1+1+1+1+1+1+1+1+1+1+1+1+1+1+1+1+1+1+1+1+1+1$

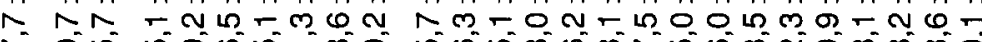

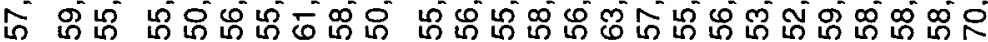

J $+1+1+1+1+1+1+1+1+1+1+1+1+1+1+1+1+1+1+1+1+1+1+1+1+1+1$ * $0 \mathrm{~N}$ n m

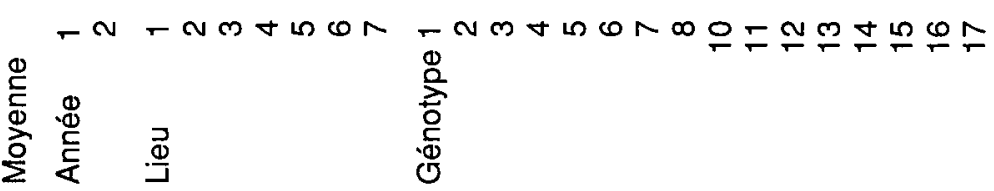


Tableau V. Corrélations entre les principaux caractères étudiés (les corrélations dont la valeur absolue est $>0,25$ sont significativement différentes de 0,0 au seuil de $1 \%$ ).

a

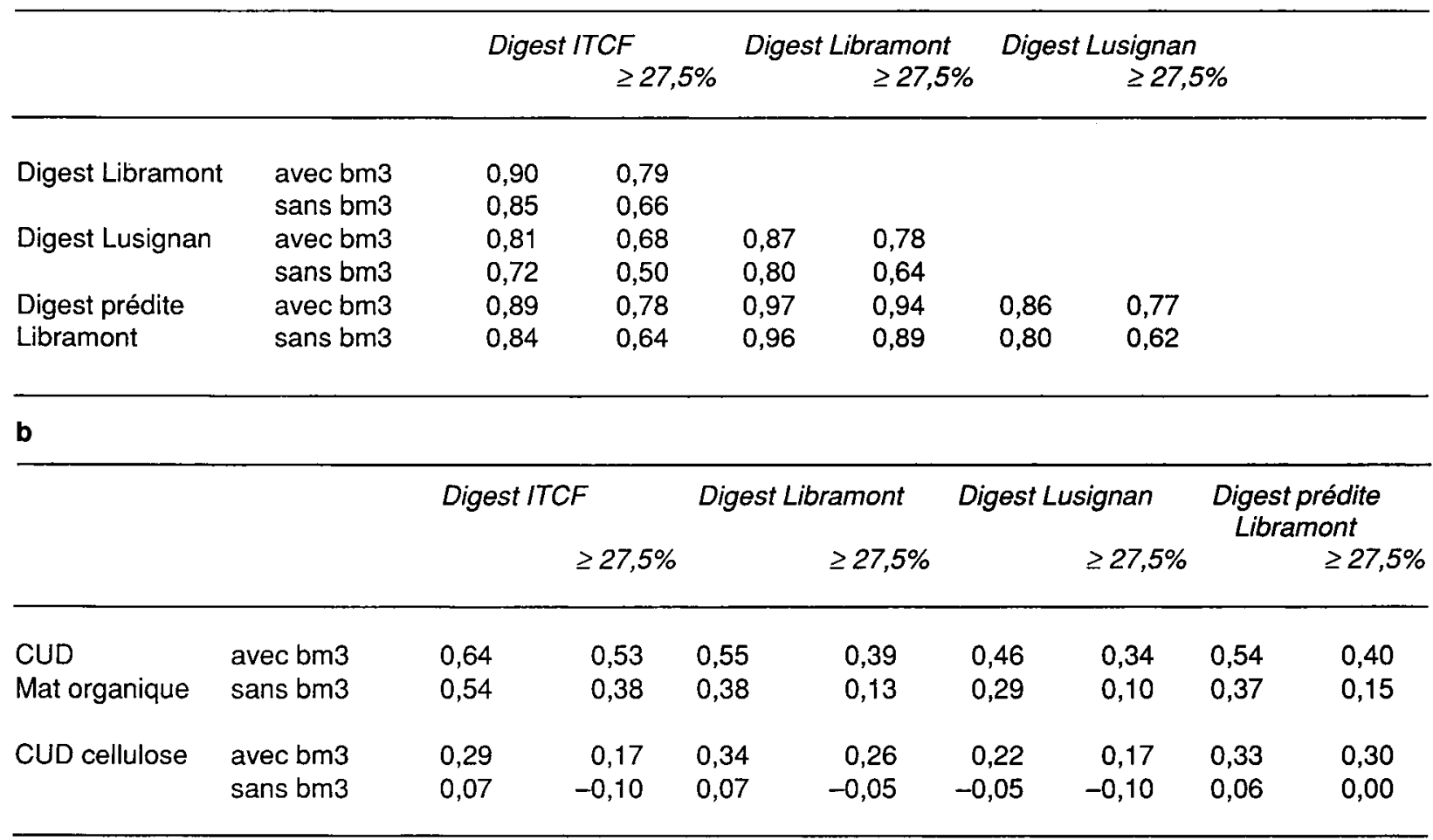

c

CUD cellulose

CUD mat organique

prédit NIRS

$\geq 27,5 \%$

$\geq 27,5 \%$

CUD mat organique

avec bm3

sans bm 3

0,69

0,63

0,53

0,90

0,85

0,88

0,80

d

\begin{tabular}{ccccc}
\hline Cellulose & NDF & ADF & Lignine & Amidon \\
$\geq 27,5 \%$ & $\geq 27,5 \%$ & $\geq 27,5 \%$ & $\geq 27,5 \%$ & $\geq 27,5 \%$ \\
\hline
\end{tabular}

CUD mat organique avec bm3 sans bm3

CUD cellulose

Digest ITCF

Digest Libramont

Digest Lusignan

Digest prédite

Libramont

CUD mat organique

prédit NIRS

$\begin{array}{rrrrrrrrrr}-0,50 & -0,48 & -0,36 & -0,37 & -0,48 & -0,49 & -0,50 & -0,40 & 0,31 & 0,24 \\ -0,49 & -0,45 & -0,34 & -0,34 & -0,45 & -0,45 & -0,36 & -0,19 & 0,26 & 0,32 \\ 0,08 & 0,15 & 0,15 & 0,14 & 0,04 & 0,07 & -0,29 & -0,25 & -0,28 & -0,27 \\ 0,18 & 0,26 & 0,17 & 0,25 & 0,24 & 0,20 & 0,15 & -0,00 & -0,29 & -0,26 \\ -0,76 & -0,68 & -0,68 & -0,59 & -0,78 & -0,73 & -0,79 & -0,66 & 0,43 & 0,24 \\ -0,81 & -0,72 & -0,73 & -0,60 & -0,78 & -0,73 & -0,72 & -0,48 & 0,56 & 0,37 \\ -0,67 & -0,52 & -0,65 & -0,52 & -0,71 & -0,57 & -0,82 & -0,73 & 0,41 & 0,14 \\ -0,74 & -0,54 & -0,72 & -0,54 & -0,77 & -0,56 & -0,76 & -0,57 & 0,57 & 0,27 \\ -0,68 & -0,54 & -0,64 & -0,47 & -0,73 & -0,62 & -0,80 & -0,72 & 0,49 & 0,22 \\ -0,73 & -0,53 & -0,68 & -0,45 & -0,77 & -0,59 & -0,73 & -0,58 & 0,63 & 0,33 \\ -0,67 & -0,52 & -0,66 & -0,52 & -0,72 & -0,57 & -0,81 & -0,71 & 0,42 & 0,12 \\ -0,74 & -0,53 & -0,74 & -0,54 & -0,78 & -0,56 & -0,74 & -0,53 & 0,59 & 0,24 \\ -0,53 & -0.55 & -0,36 & -0,35 & -0,51 & -0,52 & -0,50 & -0,41 & 0,22 & 0,25 \\ -0,55 & -6 & -0,35 & -0,32 & -0,49 & -0,49 & -0,33 & -0,16 & 0,29 & 0,36\end{array}$


élevées. Elles sont seulement comprises entre 0,50 et 0,66 quand on se limite aux hybrides normaux récoltés à un niveau de maturité suffisant; le retrait du génotype bm3 diminuant dans ce cas les liaisons de 0,13 à 0,18 points. Bien qu'il soit faible, l'effet stade mis en évidence par l'analyse de variance semble donc expliquer une part non négligeable des corrélations entre méthodes de mesures (tableau II). II y a d'ailleurs une liaison faiblement positive entre la somme de températures en base 6 entre floraison et récolte du fourrage, et le CUD de matière organique ou chacune des 3 mesures de digestibilité in vitro.

Un des objectifs de cette étude était d'estimer la liaison entre les différentes méthodes d'appréciation in vitro d'une digestibilité, et les digestibilités (ou CUD) mesurées par les moutons standards considérés comme valeur de référence. Le tableau $\mathrm{Vb}$ montre que la corrélation est relativement peu élevée entre les différentes solubilités enzymatiques et le CUD de la matière organique (entre 0,45 et 0,65 ) quand toutes les donnés sont prises en compte. Mais cette corrélation est encore plus faible (entre 0.10 et 0.40 ) quand on se limite à du matériel classique à un niveau de maturité représentatif du stade de récolte en ensilage, le retrait des fourrages bm3 étant plus marquant au niveau élevé de maturité. Une bonne partie de cette liaison est donc expliquée par la présence d'un génotype extrême, d'une part et par des effets stade maturité, d'autre part. Ces résultats confirment ceux de Lila et al (1986) qui obtenaient des corrélations similaires avec des génotypes récoltés au stade de maturité ensilage. Les liaisons entre le CUD de la cellulose et les différentes solubilités enzymatiques sont pratiquement nulles alors qu'il semble exister une certaine liaison phénotypique entre le CUD de la cellulose et CUD de la matière organique; l'analyse de variance montre que cette dernière liaison est plus liée à des effets années ou lieux qu'à des effets génotypes. Les corrélations entre les valeurs de digestibilité in vitro mesurées et celles prédites en NIRS à Libramont sont très élevées sur l'ensemble des données, mais ont tendance à diminuer quand on se limite aux échantillons dont la teneur en matière sèche est au moins égale à $27,5 \%$, en particulier quand on exclut le génotype bm3. II en est de même des corrélations entre valeurs mesurées et valeurs prédites du CUD de la matière organique. Ceci montrerait que l'équation de prédiction prend implicitement en compte des effets liés au stade de maturité et/ou au milieu, et confirme par ailleurs des observations non publiées faites par Furstoss et Lila à Lusignan sur la difficulté d'intégrer dans une même équation de prédiction des génotypes normaux et bm3. Les liaisons entre les différents constituants (cellulose, NDF, ADF, lignine) et le CUD de la matière organique sont assez faibles et négatives. Elles sont nulles ou pratiquement nulles entre ces mêmes constituants et le CUD de la cellulose. Hormis la liaison déjà citée avec le CUD de la matière organique, il apparaît en fait que le CUD de la cellulose n'est lié à aucun des autres caractères étudiés, y compris, à la teneur en cellulose, contrairement à ce que l'on observe classiquement avec d'autres espèces de plantes fourragères. Les liaisons entre ces mêmes constituants organiques et les 3 solubilités enzymatiques étudiées sont négatives, plus élevées que celles obtenues avec le CUD de la matière organique, et du même ordre de grandeur, quel que soit le constituant considéré. Mais, là encore, il semble exister un léger effet stade puisque ces corrélations ont tendance à diminuer quand on limite l'étude aux fourrages dont la teneur en matière sèche est au moins égale à $27,5 \%$. En revanche, les liaisons entre les différentes mesures de digestibilités in vitro ou in vivo et les teneurs en cellulose, NDF ou ADF, ne semblent pas ou très peu modifiées par le retrait du génotype bm3, dont la digestibilité est pourtant très différente; ce n'est pas le cas avec la teneur en lignine, pour laquelle le matériel bm3 représente une population très particulière. Les liaisons positives entre les digestibilités in vivo ou vitro et la teneur en amidon qui évolue dans le même sens que le niveau de maturité des hybrides reflètent la tendance à une certaine augmentation de la digestibilité au cours de la maturation, dans un premier temps au moins. Elles sont nettement plus élevées pour les valeurs de digestibilité obtenues par les méthodes in vitro, que pour celles obtenues avec le CUD de la matière organique, pour l'ensemble des échantillons. Quand on se limite aux échantillons dont la teneur en matière sèche du distribué est au moins égale à $27,5 \%$, ces corrélations diminuent nettement pour les valeurs in vitro, alors qu'elles sont stables avec le CUD de la matière organique. Par ailleurs, les liaisons entre digestibilité et teneur en amidon ou en constituants pariétaux sont très généralement plus élevées en l'absence du génotype bm3. Enfin, les corrélations entre les digestibilités et les teneurs en constituants pariétaux sont, en règle générale, supérieures aux corrélations entre les digestibilités et les teneurs en amidon. 
La teneur en grain n'est, classiquement, qu'une des composantes de la digestibilité du maïs ensilage.

\section{CONCLUSIONS}

Des conclusions peuvent être dégagées à plusieurs niveaux.

L'effet du niveau de maturité sur la CUD de la matière organique ou de la cellulose est faible, ce qui confirme les observations antérieures de Andrieu et Demarquilly (1974); il en est de même de cet effet sur les valeurs de solubilités enzymatiques. Mais il y a toutefois une tendance à l'augmentation de la valeur alimentaire avec le niveau de maturité, dans l'intervalle de teneur en matière sèche étudié. II y a aussi un effet sur la variabilité phénotypique observée avec une plus grande variation des facteurs considérés à des niveaux de maturité plus élevés, ainsi que sur la qualité des mesures avec des écarts types résiduels plus faibles sur le sous-ensemble des maïs fourrage les plus mûrs.

Les 3 méthodes de solubilité enzymatique utilisées conduisent à des résultats différents. La méthode de Libramont demeure la plus discriminante (niveau du test de Fisher de l'effet génotype), quelles que soient les teneurs en matière sèche des fourrages. La méthode de Lusignan lui apparaît équivalente sur les fourrages dont la teneur en matière sèche est au moins égale à $27,5 \%$, ce qui correspond d'ailleurs aux teneurs des ensilages sur lesquels elle a été mise au point. La méthode mise en œuvre à l'ITCF est équivalente à celle de Libramont sur l'ensemble des fourrages, mais paraît moins bien différencier les génotypes quand on se limite aux maïs ayant une teneur en matière sèche au moins égale à $27,5 \%$. Les inversions de classement sur le matériel intermédiaire conduisent à de faibles corrélations entre les méthodes, alors qu'il semble bien que les génotypes extrêmes soient reconnus. Sur la base génétique étudiée, la variabilité pour les caractères liés à la valeur alimentaire varie avec la précocité des génotypes, mais n'est pas seulement un effet de la précocité en soi.

Avec la collection de maïs fourrage étudiée ici, la valeur alimentaire estimée par les moutons standards apparaît peu corrélée avec les mesures in vitro, en particulier quand on exclut le génotype bm3, la méthode développée par Aufrère (1982) apparaissant toutefois un peu plus proche des résultats obtenus sur les moutons. À ce stade, il peut donc sembler difficile de conclure sur la pertinence de ces méthodes enzymatiques pour la prédiction de digestibilité, voire de la valeur alimentaire pour des gros ruminants, de différents maïs fourrage. Toutefois, ces travaux ont permis d'approcher certaines corrélations entre des critères de constitution biochimique ou pariétale et des valeurs de digestibilité. Ceci permet de conforter des possibilités de prédiction de la digestibilité et/ou de la valeur énergétique d'un ensilage de maĩs à partir d'un ensemble de données analytiques, incluant ou non une digestibilié in vitro (Andrieu, 1990; Rivière, 1990a). En revanche, il n'est pas possible de tenter de prédire des différences entre génotypes à partir de différences mesurées entre stades ou entre lieux.

Dans une optique de sélection de maïs ensilage améliorés pour la valeur alimentaire, ces travaux montrent qu'il y a des méthodes in vitro utilisables en grande série, en particulier après étalonnage dans le proche infrarouge, qui permettent de mettre en évidence une variabilité génétique significative pour les caractéristiques liées à la valeur alimentaire du maiis fourrage. Si les corrélations entre ces méthodes in vitro et le CUD de la matière organique apparaissent faibles, c'est aussi parce que les solubilités enzymatiques telles qu'elles sont pratiquées n'expriment pas la même variation que celle qui est susceptible d'être révélée par les moutons standards. Ces valeurs enzymatiques, après une solubilisation en principe complète des composés biologiquement digérables, expriment plus un potentiel maximal de digestibilité, réalisé ou non par des moutons à l'entretien. Ces résultats rejoignent ceux de Barber et al (1990) qui, avec 3 populations différentes d'ensilage d'herbe, obtiennent 3 régressions différentes entre le CUD de la matière organique, et une solubilité enzymatique, alors qu'une seule régression rend compte de la variation entre ce même CUD de la matière organique et une digestibilité in sacco avec des animaux fistulés. Cela nous conduit à une attitude critique vis-à-vis de ces outils d'évaluation de la digestibilité. L'outil mouton standard a clairement démontré son efficacité au niveau interspécifique ou interstade. Ainsi, avec différentes graminées et légumineuses pérennes récoltées à différents stades, les digestibilités de la matière organique mesurées sur moutons sont en liaison étroite avec celles observées sur bovins, avec une valeur supérieure de 5,9 points en moyenne chez les gros ruminants (Chenost 
et Martin-Rosset, 1985). Sur des maïs récoltés entre 18,6 et $48,3 \%$ de matière sèche, la digestibilité de l'énergie et celle de la matière sèche n'étaient pas significativement différentes entre moutons et vaches laitières Holstein (Nakui et al, 1982). Enfin, sur des ensilages de maiis dont la teneur en grain variait entre 18 et $33 \%$, les valeurs énergétiques UFL prédites à partir des digestibilités faites sur moutons standards sont en assez bon accord avec les performances observées, sur vaches laitières, avec toutefois une surestimation irrégulière de cette valeur énergétique liée en particulier à la teneur en concentrés des rations (Rivière, 1990b). Mais cet outil mouton standard n'est pas forcément celui qui est le mieux adapté à prédire des différences entre génotypes d'une manière espèce, dans une gamme relativement étroite de niveau de maturité, pour des gros ruminants alimentés à des niveaux de 2 ou 3 fois les besoins de la maintenance; ce qui est précisément l'objectif de la recherche sur maïs ensilage. Colucci et al (1989) mettent ainsi en évidence des différences de digestibilités de certains éléments entre moutons et vaches laitières, à des niveaux élevés d'ingestion. Parallèlement, les solubilités enzymatiques actuellement utilisées ne donnent pas d'indication sur les aspects de facilité ou de rapidité de dégradation des constituants pariétaux, et ne sont donc pas optimales; il serait également important de trouver le moyen, avec par exemple des cinétiques ou des digestions cellulosiques pendant des durées plus courtes, d'inclure à l'aspect prévision de la digestibilité, l'aspect prévision des quantités ingérées, qui n'est pas indépendant de la facilité de dégradation. L'objectif du sélectionneur est d'avoir une méthode in vitro lui permettant de reconnaître et de trier les génotypes de maïs susceptibles d'apporter, soit une amélioration des performances zootechniques de ruminants à haut potentiel, soit une diminution de leurs performances en raison d'une dérive vers des plantes moins digestibles au cours de travaux de sélection pour la productivité en biomasse ou la résistance aux verses, que cette méthode in vitro s'avère ou non statistiquement bien corrélée à une mesure de digestibilité sur mouton standard. Avec les résultats dont nous disposons maintenant, il semble que des solubilités enzymatiques puissent être utilisées efficacement en sélection. Elles doivent permettre d'éliminer les génotypes à faible digestibilité. Elles doivent également permettre de trier des génotypes potentiellement supérieurs, dont la valeur devra être contrôlée sur moutons et/ou sur gros ruminants tant que la validité de la meilleure de ces méthodes enzymatiques, ou de méthodes enzymatiques améliorées n'aura pas été complètement confirmée, comme prédicteur pertinent d'une production de lait ou de viande. À ce titre, les expérimentations déjà réalisées sur des gros ruminants avec des génotypes connus pour leur bonne, ou respectivement, leur faible digestibilité in vitro sont peu nombreuses. Gielen et al (1988) et Clinquart et al (1990) montrent une très bonne liaison entre les mesures de digestibilités mesurées sur taurillons et les solubilités enzymatiques faites selon la méthode de $\mathrm{Li}$ bramont. Les croissances de taurillons vont dans le sens attendu après les mesures de digestibilité, avec un risque de biais lié à une teneur en matière sèche plus élevée pour un des génotypes, et la sous-estimation de la valeur alimentaire d'un autre génotype qui s'avère mieux ingéré. En revanche, Barrière et Émile (1990) ne retrouvent pas, avec la solubilité enzymatique selon la méthode de Lusignan, la meilleure aptitude à la production laitière d'un hybride ancien comparé à un hybride moderne. L'étude, sur une large base génétique de niveau élite, de la variabilité génétique de la digestibilité in vitro, puis celle de la digestibilité ou de l'efficacité in vivo de génotypes reconnus différents, permettra de conclure sur la pertinence de ces solubilités enzymatiques pour l'amélioration des maïs fourrage. L'héritabilité de la digestibilité in vitro, souvent de même ordre que celle de la productivité en plante entière et pouvant atteindre des valeurs supérieures à 0,8 (Dhillon et al, 1990) montre que des gains notables sont possibles à court ou moyen terme sur ce critère dont il faudra de même vérifier l'efficacité sur animaux. Enfin, des progrès dans la connaissance de la variabilité des lignines et maillages entre lignines et celluloses sont sans doute parmi les composantes indispensables à des avancées significatives, en terme de compréhension des facteurs de digestibilité et d'ingestibilité des plantes de maïs ensilage et donc, des possibilités efficaces de prédiction.

\section{REMERCIEMENTS}

Nous remercions les membres du Club digestibilité ainsi que les expérimentateurs de chacune des unités qui ont permis le financement et la réalisation de ces travaux. 


\section{RÉFÉRENCES}

Andrieu J (1990) Compte rendu des essais du groupe de travail "Club digestibilité". INRA, document ronéoté

Andrieu J, Demarquilly C (1974) Valeur alimentaire du maïs fourrage. II. Influence du stade de végétation, de la variété, du peuplement, de l'enrichissement en épis et de l'addition d'urée sur la digestibilité et l'ingestibilité de l'ensilage de maïs. Ann Zootech 23, 125

Andrieu J, Demarquilly C, Sauvant D (1988) Tables de valeur nutritive des aliments. In: Alimentation des bovins, ovins, caprins (sous la direction de $\mathrm{R}$ Jarrige) INRA, 356-464

Aufrère J (1982) Étude de la prévision de la digestibilité des fourrages par une méthode enzymatique. Ann Zootech 31, 111-130

Barber GD, Givens DI, Kridis MS, Offer NW, Murray I (1990) Prediction of the organic matter digestibility of grass silage. Anim Feed Sci Technol 28, 115-128

Barrière Y, Émile JC (1990) Effet des teneurs en grains et de la variabilité génétique sur la valeur énergétique du maïs ensilage mesurée par des vaches laitières. Agronomie 10, 201-212

Barrière Y, Émile JC, Traineau R (1988) Energy value of silage maize and zootechnical performances. Preliminary report of the study of genetic variability. In: Proceedings, quality of silage maize, digestibility and zootechnical performance. Gembloux, Belgique, 105-118

Biston R, Dardenne P (1988) Applying NIRS to measure the silage maize in vitro and in vivo digestibility. In: Proceedings quality of silage maize, digestibility and zootechnical performance. Gembloux, Belgique, 56-57

Chenost M, Martin-Rosset W (1985) Comparaison entre espèces (mouton, cheval, bovin) de la digestibilité et des quantités ingérées des fourrages verts. Ann Zootech 34, 291-312

Clinquart A, Gielen M, Dufrasne I, Istasse L, Van Eenaene C, Bienfait JM (1990) Comparaison d'ensilages de maïs de quatre variétés différentes dans l'engraissement des taurillons. Univ Liège, document ronéoté, $3 p$
Colucci PE, MacLeod GK, Grovum WL, Cahill LW, McMillan I (1989) Comparative digestion in sheep and cattle fed different forage concentrate ratios at high and low intakes. J Dairy Sci 72, 1774-1785

Deinum B, Struik PC (1985) Improving the nutritive value of forage maize. In : Proc 13th Congr maize and sorghum section, Eucarpia. Wageningen, Pays Bas, 9-12 Septembre 1985, 77-90

Dhillon BS, Paul C, Zimmer E, Gurrath PA, Klein D, Polmer WG (1990) Variation and covariation in stover digestibility traits in diallel crosses of maize. Crop Sci 30, 931-936

Gielen M, Dufrasne I, Istasse L, Bienfait JM, Biston R (1988) Relationship between digestibility of maize silage and performances of growing fattering bulls. In: Proceedings quality of silage maize, digestibility and zootechnical performance. Gembloux, Belgique, 119-127

Jarrige R (ed) (1988) Alimentation des bovins, ovins et caprins. INRA, $471 \mathrm{p}$

Lila M, Barrière Y, Traineau R (1986) Mise au point et étude d'un test enzymatique de la digestibilité de fourrages pauvres ou riches en amidon. Agronomie $6,285-291$

Martens $H$, Jensen SA (1982) Partial least squares regression: a new two stage NIR calibration method. In: Proc 7th World Cereal Bread Congr (Holas, Kratochvil, eds) Elsevier, Amsterdam, 607-647

Nakui T, Iwasaki K, Hayakawa M (1982) The comparison of dairy cows with sheep in the digestibility of whole crop corn silage prepared from identical origins. J Jpn Grassl Sci 28, 111-116

Rivière F (1990a) Compte rendu des essais du groupe de travail "Club digestibilité". ITCF, document ronéoté

Rivière $F$ (1990b) Valeur nutritive et valorisation de l'ensilage de maïs par les vaches laitières. $\mathrm{In}$ : $\mathrm{Col}$ loque maïs ensilage et céréales pour les ruminants. ITCF-AGPM, Angers 4 avril 1990, 44-56

Ronsin T, Femenias N (1990) Use of NIRS determination of quality in a silage maize breeding program. 3rd Int Conf on near infrared spectroscopy. Bruxelles, Belgique, juin 25-29

Tilley JMA, Terry RA (1963) A two stage technique for the in vitro digestion of forage crops. $J$ Br Grass/ Soc 18, 104-111 